\title{
Rotational Acceleration Measurements - Evaluating Helmet Protection
}

\author{
M. Kis, F. Saunders, M.W. ten Hove, J.R. Leslie
}

\begin{abstract}
Purpose: Current helmet testing standards do not address the rotational components of an impact to the head. We describe a new testing paradigm used to measure the rotational acceleration of a headform and a protective helmet following an impact to the head in the horizontal plane. This impact simulation allows for the testing of currently available head protection devices in conditions thought to be important for the generation of cerebral concussion. The degree to which a particular helmet dampens rotational acceleration, and thus protects against concussion, can be assessed. Methods: Our testing device consists of a pneumatic piston that provides a measured impact to a standard headform. Four different helmets were tested using the described paradigm. Results: Acceleration curves for each helmet and the corresponding headform are presented. Conclusions: Clear differences in rotational acceleration were demonstrated. Possible avenues of further investigation are discussed.
\end{abstract}

RÉSUMÉ: Mesure de l'accélération rotatoire - Évaluation de la protection conférée par un casque. Objectif : Les standards d'évaluation actuels des casques protecteurs ne tiennent pas compte des composantes rotatoires d'un impact à la tête. Nous décrivons un nouveau paradigme d'évaluation utilisé pour mesurer l'accélération rotatoire sur une tête de mannequin et un casque protecteur suite à un impact à la tête dans le plan horizontal. Cette simulation d'impact permet l'évaluation des casques protecteurs actuellement disponibles sur le marché dans des conditions qui semblent importantes dans la genèse des commotions cérébrales. Le degré d'atténuation de l'accélération rotatoire d'un casque et donc de protection contre la commotion cérébrale peut ainsi être évalué. Méthodes : Notre instrument d'évaluation est composé d'un piston pneumatique qui fournit une mesure d'impact à une tête de mannequin standard. Quatre casques différents ont été évalués au moyen de cet instrument. Résultats : Nous présentons les courbes d'accélération pour chaque casque et la tête de mannequin correspondante. Conclusions : Nous démontrons qu'il existe des différences importantes dans l'accélération rotatoire et nous discutons des pistes à explorer davantage.

Can. J. Neurol. Sci. 2004; 31: 499-503

The mechanism and prevention of concussion have been extensively investigated in the last decade. Concussions have both immediate and long-term psychological and intellectual effects. ${ }^{1-3}$ It has been shown that the effects of each concussion may be additive ${ }^{4}$ and that a second concussion, sustained before the effects of the first injury are over, may be fatal. ${ }^{5}$ Amateur and professional sports are seen to be becoming more violent and concussions are being recognized more frequently than they have been in the past. ${ }^{6}$

Unfortunately, there is no effective therapy for the concussed brain; the only worthwhile approach is prevention. ${ }^{7}$ Rules have changed in some sports in the hope of decreasing concussion frequency. Players, coaches, and officials have been educated about the types of contact that may lead to concussion and the importance of refraining from further play until all of the symptoms of concussion have resolved. Player equipment and sports facilities have also been modified in an attempt to provide maximum protection for the athlete. All hockey players are now required to wear helmets and this has been shown to decrease head injury. ${ }^{8}$ However, despite these efforts, the frequency of reported concussions continues to increase. ${ }^{9}$

Although the exact nature of concussion is still unclear, research has shown that exacerbating factors are a mobile head, ${ }^{10}$ and high rotational accelerations. ${ }^{10-12}$ Current techniques for helmet testing do not attempt to measure the helmet's effect on absorbing rotational acceleration. ${ }^{13,14}$ We designed a testing paradigm to specifically address this important biomechanical aspect of concussion.

From the Department of Neurosurgery, (MK), Dalhousie University, Halifax, NS; Department of Neurosurgery, (FS); Department of Neuro-Ophthalmology, (MWtH); Department of Physics, (JRL); Queen's University, Kingston, Ontario Canada. ReCEIVEd November 19, 2003. ACCEPTED INFINALFORM June 23, 2004. Reprint requests to: Fraser Saunders, 95 King Street E., Kingston, ON, K7L2Z7 Canada. 


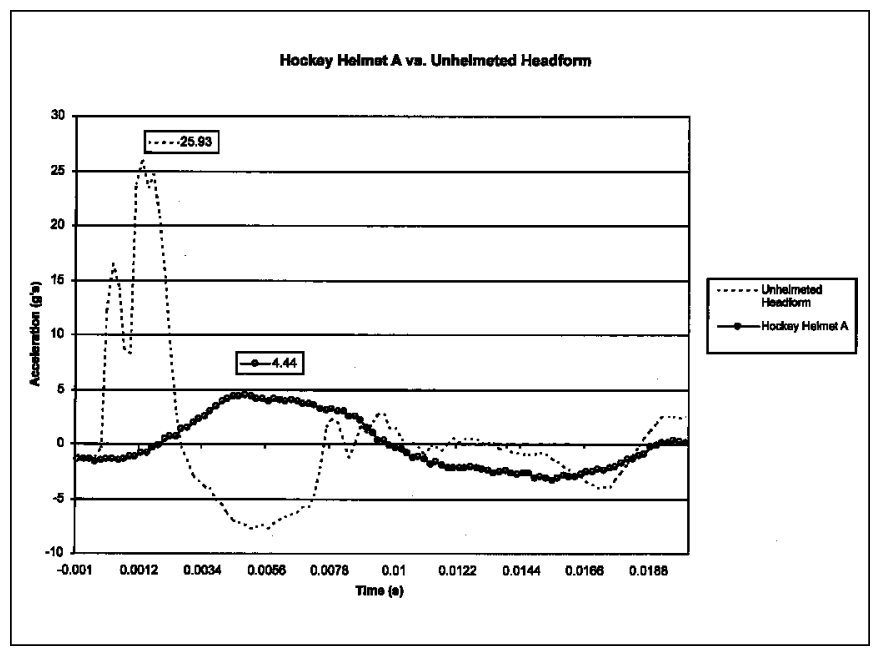

Figure 1: Hockey helmet A vs. unhelmeted headform

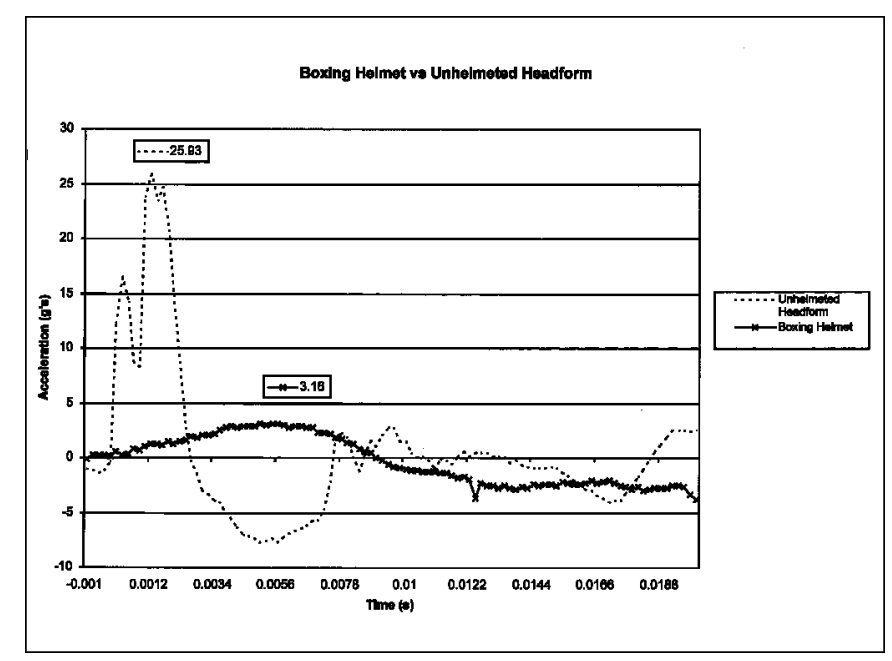

Figure 3: Boxing Helmet vs Unhelmeted Headform

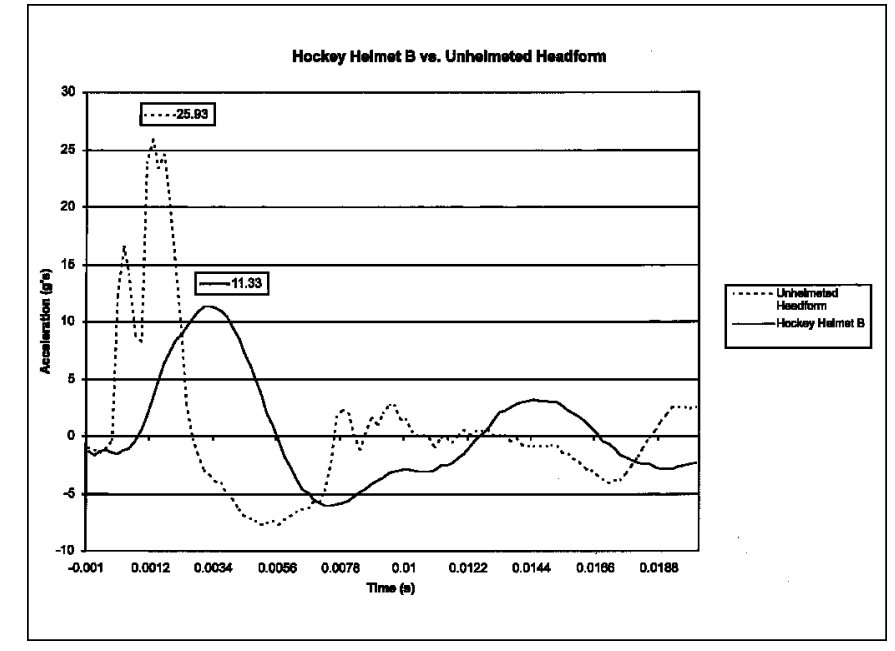

Figure 2: Hockey Helmet B vs. Unhelmeted Headform

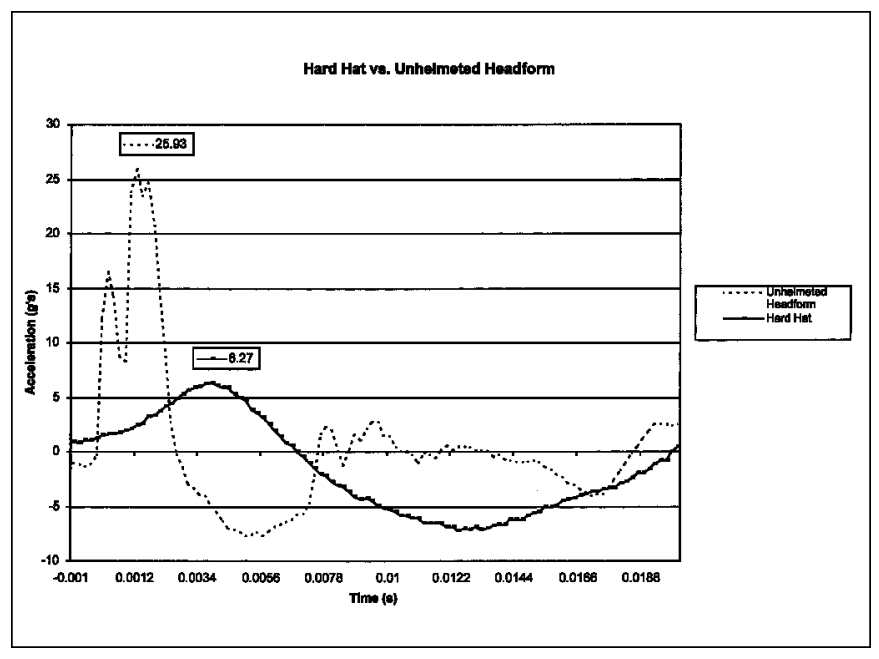

Figure 4: Hard Hat vs. Unhelmeted Headform

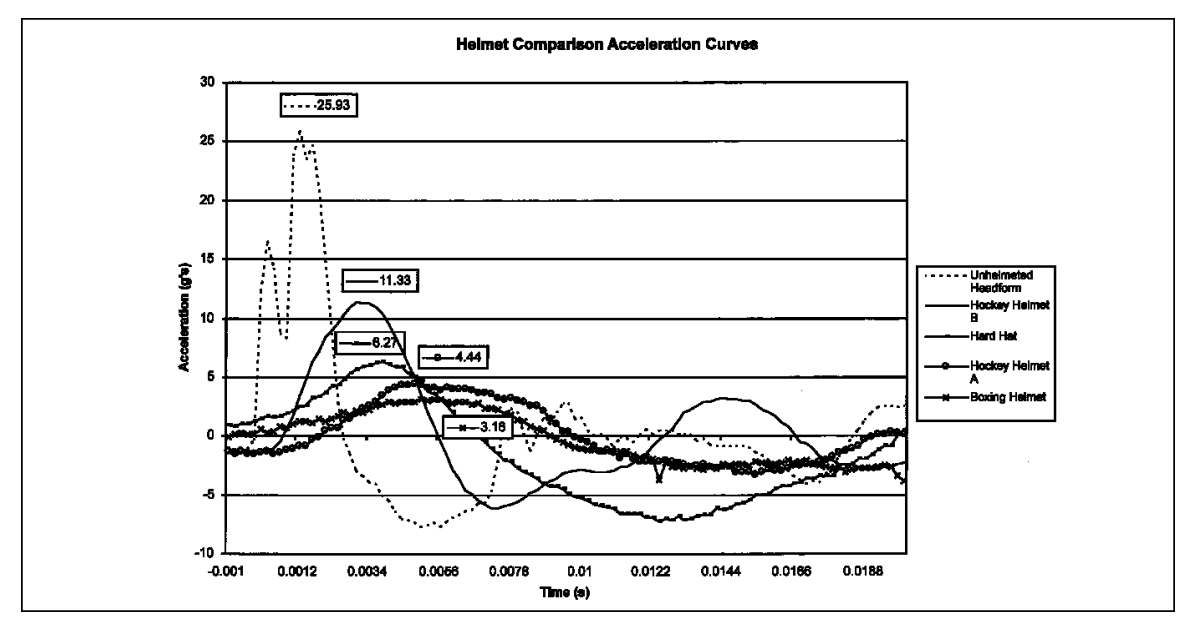

Figure 5: Helmet Comparison Acceleration Curves 


\section{METHODS}

The testing paradigm we employed uses an impact force to induce a rotational acceleration in the horizontal plane. The test "subject" was an ISO standard anthropometric humanoid headform composed of a magnesium-aluminum alloy. A size ' $\mathrm{J}$ ' headform was used, corresponding to an average head size with a circumference of $57 \mathrm{~cm}$. The head was mounted on a flat wooden surface using a brass bearing with a diameter of 1.25 inches. The axial play of the bearing was 0.0005 inches, that in effect limited the motion of the head to rotation in the horizontal plane. This was done in order to isolate rotational velocity and acceleration of the headform.

Above the headform spanned a circular stainless steel arc that was fastened to the support surface. A PARKER pneumatic cylinder with a 1.06 inch bore was mounted to the arc frame. The cylinder contained a stainless steel piston that could be propelled forward by air pressure. To the end of the piston was attached a lead disk that was used as the impacting surface. The motion of the piston was controlled by a dual action unrestricted flow valve which, when activated using an electrical switch, would cause the piston to move forward, impact the object, and retract immediately. The piston stroke length was 4 inches.

The energy produced by the pneumatic cylinder depended upon the air pressure used to propel the piston and the length along the piston stroke that the impact occurred. By keeping these two variables constant, a consistent force of impact could be ensured. The air pressure used for each impact was $125 \mathrm{psi}$ and the impacts occurred at 3.625 inches along the stroke length. The force produced by the piston was $492.7 \mathrm{~N}$. The energy conferred to the system was $44.7 \mathrm{~J}$. These values, and the subsequent accelerations produced, are consistent with concussion level forces in prior experimental situations. ${ }^{14,15}$

The compressed air was produced by a portable compressor and the electrical switch was powered by a $12 \mathrm{~V}$ transformer. The mounting of the cylinder was such that it could be moved up and down along the length of the arc as well as pivot \pm 30 degrees in place. By turning the head 180 degrees, impacts could be simulated on opposite sides. The arc itself could be moved

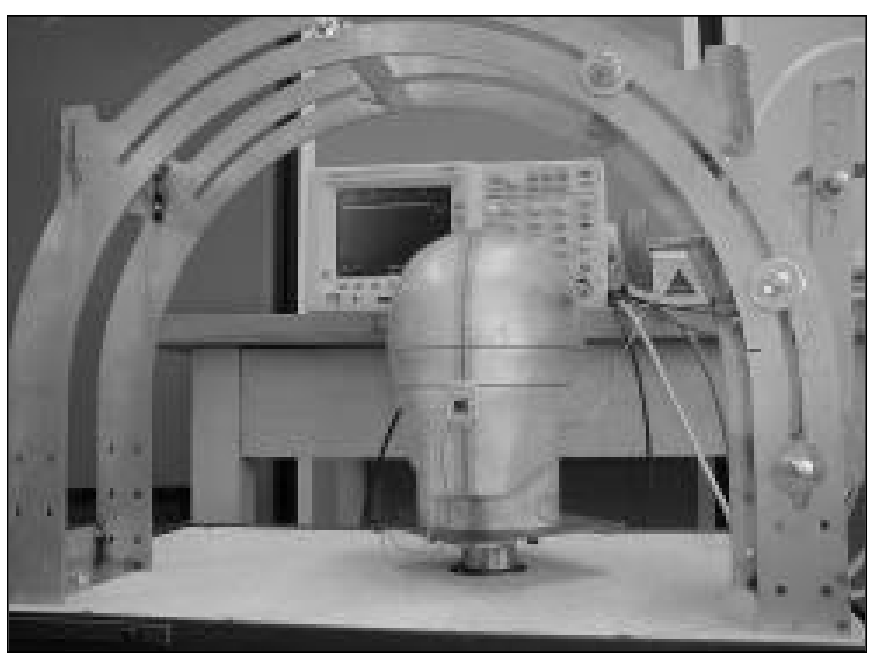

Figure 6: Head form and impact apparatus laterally along the side of the wooden surface. The ability to adjust all these factors made it possible to simulate consistent impacts in any of the three dimensions on the helmet or headform surface.

To measure the rotational accelerations of the helmet relative to the headform, one uniaxial accelerometer was mounted on the headform and one was mounted on the helmet at the most anterior location possible. All impacts occurred on the anterolateral surface, simulating a blow to the anterior temporal area. Every effort was made to standardize the impact location for all five trials of each helmet and between the various helmet types. Previous experiments had shown that five trials were sufficient to acquire an average acceleration curve. The acceleration measurements were obtained by the accelerometer transmitting a voltage change that was recorded by a digital oscilloscope. Two waveforms were recorded for each impact: one for the helmet and one for the headform. The current paper is concerned only with the accelerations experienced by the headform, both with and without headgear. The data gathered regarding helmet motion will therefore not be presented here.

The initial series of impacts were on an unprotected headform, in order to establish a baseline and a basis of comparison for the degree of helmet protection. The accelerometer was on the most frontal aspect of the headform. The second series of impacts used two different models of hockey helmets, a boxing helmet and a construction safety helmet. The accelerometer in each case was placed on the most anterior aspect of the headform. Each impact test consisted of five impacts averaged into an acceleration curve. Helmets were fitted according to the manufacturers'recommendations.

\section{Results}

Figure 1 shows the acceleration profile obtained for Hockey helmet A. Figure 2 shows the accelerations measured with Hockey helmet B. Figure 3 shows the reduction of rotational acceleration afforded by the boxing helmet, and Figure 4 shows the results obtained with the construction helmet. A composite graph of all helmets tested is shown in Figure 5. All figures also illustrate the unhelmeted headform acceleration curve for comparison.

\section{Discussion}

Multiple theories for the mechanism of concussion exist. ${ }^{16}$ The oldest of these theories is the vascular hypothesis of concussion. The loss of consciousness and the other neuropsychological changes that may accompany a concussion are postulated to be due to transient cerebral ischemia. Although popular for many years, this theory does not account for the immediate onset of symptoms, moreover, recent studies of cerebral energy production in concussed rat brains have failed to show any decrease in metabolism. ${ }^{17}$ Some researchers believe the vascular theory may explain some of the long-term side effects of concussion such as amnesia. ${ }^{16}$

The reticular theory argues that the symptoms of concussion are the result of a disturbance in the brainstem reticular formation following a traumatic event. Since damage to the brainstem can cause somnolence, stupor, coma or even death, it was argued that a less severe insult could cause a transient 
disturbance in function. Multiple mechanisms have been proposed to account for such transient impairment. Elastic distortion, stretching, shearing, disruption due to intracranial pressure gradients and/or rotational accelerations, and induced release of acetylcholine have all been postulated as mechanisms by which the reticular formation in the brainstem is damaged.

In the 1970 s, Ommaya and Gennarelli ${ }^{10}$ postulated the centripetal hypothesis. Their theory was founded on a more refined understanding of the biomechanics of concussion. ${ }^{12,18}$ They theorized that rotational forces play a key role in concussion and that concussion occurs as a result of shearing strains and stresses between various brain regions of differing tissue densities. Such rotational velocities and stresses are greatest at the peripheral interfaces between the superficial gray matter and the deeper white matter.

The pontine cholinergic hypothesis, formulated in the 1980s by Hayes et al, ${ }^{19}$ is similar to the reticular theory in that it too points to a disturbance in the reticular formation as the main factor in concussion. It postulates a mechanical disruption of the brainstem during a concussive event, which activates an inhibitory cascade involving release of the neurotransmitter acetylcholine. This activation of an "inhibitory system" is what differentiates the pontine cholinergic theory from the reticular theory. The latter postulates the depression of an "activating system" rather than the activation of an "inhibitory system" to account for the concussive symptoms. Proponents cite the beneficial effect of anticholinergics administered to a concussed patient, as well as the detrimental, concussion-like effects that can be induced by introducing cholinergic agonists into the brains of test animals. ${ }^{16}$

The convulsive theory argues that the symptoms of a concussion share many similarities with epileptic seizures. It postulates that a concussive blow sets up complex movements of the brain within the cranium. Collisions occur between various brain regions and the skull. Such collisions create mechanically induced depolarisations of the cortex creating a synchronised discharge of cortical neurons. Evidence for this theory comes from various EEG and other neurophysiological studies. ${ }^{16}$

Many laboratory experiments have sought to simulate concussions. They have utilized both cadaveric and animal models. The latter have included both higher and lower mammals, such as rats, mice, cats, ferrets, pigs, squirrel monkeys, baboons and chimpanzees. Compressive experiments involve using some mechanism to directly compress the brain or dura. While these compression studies do not seek to be accurate in reproducing the precise mechanism of concussive injury, in that the force is applied directly to the dura and not transmitted through the skull, they do allow experimentation with a fixed head. This makes instrumentation and data gathering such as EEGs easier to accomplish. ${ }^{16}$ Acceleration models use a mobile head but have to contend with the difficulties inherent in gathering information from a moving target. There are two general methods of delivering accelerative/decelerative injury in acceleration models: by impact or by impulse. Impact involves a blow making direct contact with the head, while impulse involves setting the head in motion using an accelerative force without directly striking it. ${ }^{16}$

When using a biological model to test concussions, an impact injury, in addition to providing acceleration/deceleration, also sets up pressure waves within the cranial vault. ${ }^{20}$ This possible confounding factor can be avoided by using a nonimpact, purely impulse paradigm. The most sophisticated and quoted of these is the "PENN II" device by Ommaya and Gennarelli. ${ }^{10}$

From these experimental models it appears that a mobile head is more susceptible to concussion than a fixed head. It also appears that rotational acceleration is more damaging than linear accelerating forces. ${ }^{10-12}$ Although the exact mechanism of concussion is not fully understood, it appears that traumatic force is required and that rotational force is more important than translational force. ${ }^{10,11}$

Helmets have been designed to protect the cranial vault from skull fracture and major injury. Their efficacy is welldocumented. ${ }^{8}$ However it has not been documented how much they aid in the prevention of specific concussive injury.

Helmet performance, or protection, as it is currently evaluated, involves an assessment of linear acceleration protection. The testing paradigm uses experimental deceleration through an impact. The regulatory standards involve linear drops of 6-9 ft. of a helmeted metal headform onto a flat metal surface, while recording the acceleration of the centre of mass of the headform at impact. ${ }^{13}$ The metal headform is not designed to simulate the skull-brain interface. It does not contain a simulated brain component such that there are no confounding variables of impact-generated intracranial pressure gradients to contend with. ${ }^{16}$

Many head injury research protocols exist that measure acceleration changes. However, the accelerometers are frequently placed within the head or at the centre of gravity, which is not the place of maximal rotational acceleration potential. ${ }^{8}$ As such, the role of purely rotational forces in impact models has not been isolated.

Our device simulated the rotational acceleration of a helmeted headform. Every effort was made to design the testing paradigm such that it simulated currently accepted common mechanisms for the generation of cerebral concussions. Current helmet standards generally utilize impact energies between 40-150 J. ${ }^{13,15}$ We chose to use a relatively low impact force of $44.7 \mathrm{~J}$. We chose a location of impact that caused maximal rotational acceleration. Impacts in the temporal area that result in horizontal rotation of the head have been shown to be particularly prone to generating concussions. $^{10,15}$

Current helmet testing does not address these concerns. Linear deceleration recordings from the centre of the head do not appear to match the research findings that are important in concussive injury. ${ }^{3,10,11}$ It is, however, important to note the recent work of Pellman and colleagues ${ }^{21-23}$ in investigating the biomechanics of head injuries leading to concussion in the National Football League. They utilized video footage of actual game-time head injuries and, using sophisticated image analysis techniques, reconstructed the impacts in the laboratory. Their results clearly illustrate that impacts on the facemask area were most likely to cause concussions. They state "impacts to the facemask generally twist the head while accelerating it". ${ }^{23}$ This type of impact clearly has a component of rotational acceleration associated with it. They found, however, that, contrary to earlier studies, translational acceleration measurements were more closely related to clinical concussion than rotational accelerations. It was also found that changes in translational 
acceleration were reflected in similar changes to rotational acceleration. Their conclusion was that helmet testing need only involve translational acceleration measurements.

Because the brain mechanisms for concussion are not precisely defined, we believe that translational and rotational acceleration must be separated and studied independently for further concussion understanding and helmet design. We developed a testing technique that evaluates relatively low force rotational impacts. The degree of rotational acceleration protection can be easily established for any helmet design and fitting characteristic. It is our hope that this testing paradigm will lead to further improvement in the design, construction, and fitting of helmets to maximize protection against rotational injury. It is apparent from Figure 5 that while all helmets dampen rotational acceleration, they do so to different degrees. The current experiment was only designed to investigate whether differences exist between rotational acceleration characteristics of various helmets. Further study is necessary to understand the reasons behind these differences. The authors plan to use this testing technique using a wide range of impact forces and locations to more completely characterize the rotational protective abilities of helmets.

\section{ACKNOWLEDGEMENTS}

The authors thank Mihaly Kis Sr. for his invaluable help in the construction of the impactor prototype.

\section{REFERENCES}

1. Bohnen N, Jolles J, Twijnstra A. Neuropsychological deficits in patients with persistent symptoms six months after mild head injury. Neurosurgery 1992; 30(5): 692-695.

2. Barth JT, Macciocchi SN, Giordani B, et al. Neuropsychological sequelae of minor head injury. Neurosurgery 1983; 13:529-533.

3. Rimel RW, Geordani B, Barth JT, et al. Disability caused by minor head injury. Neurosurgery 1981; 9: 221-228.

4. Erlanger DM, Kutner KC, Barth JT, et. al. Neuropsychology of sports related head injury: Dementia Pugilistica to post concussion syndrome. Clin Neuropsychol 1999; 13(2): 193-209.

5. Cantu RC. Second-impact syndrome. Clin Sports Med 1998; 1: 37-44.

6. Cantu RC. Head injuries in sport. Br J Sports Med 1996; 30: 189-196.
7. McCrory P. New treatments for concussion: the next millennium beckons. Clin J Sport Med 2001; 11: 190-193.

8. Henderson M. The effectiveness of bicycle helmets: a review. Paper presented at Motor Accidents Authority of New South Wales, Australia (Bicycle Helmet Safety Institute), ISBN 0 T310 64356. 1995.

9. Covassin T, Swanik CB, Sachs ML. Epidemiological considerations of concussions among intercollegiate athletes. Appl Neuropsychol 2003; 10(1): 12-22.

10. Gennarelli TA, Thibault LE, Adams JH, et al. Diffuse axonal injury and traumatic coma in the primate. Ann Neurol 1982; 12: 564-574.

11. Ommaya AK, Gennarelli TA. Cerebral concussion and traumatic unconsciousness: correlation of experimental and clinical observations on blunt head injuries. Brain 1974; 97: 633-654.

12. Holbourn AHS. Mechanics of head injuries. Lancet 1943; 2: 438441.

13. Halstead PD. Performance testing updates in head, face and eye protection. J Athletic Training 2001; 36(3): 322-327.

14. Halstead PD, Alexander CF, Cook EM, Drew RC. Hockey headgear and the adequacy of current designs and standards. In: Ashare $\mathrm{AB},(\mathrm{Ed})$. Safety in Ice Hockey. American Society for Testing and Materials. Philadelphia: 1998:93-101.

15. McIntosh A, McCrory P, Comerford J. The dynamics of concussive head impacts in rugby and Australian rules football. Med Sci Sports Exerc 2000; 32(12): 1980-1984.

16. Shaw NA. The neurophysiology of concussion. Prog Neurobiol 2002; 67: 281-344.

17. Nilson B, Ponten U. Experimental head injury in the rat. Part 2. Regional brain energy metabolism in concussive trauma. J Neurosurg 1977; 47:252-261.

18. Symonds CP. Concussion and its sequelae. Lancet 1962; 1:1-5.

19. Hayes RL, Lyeth BG, Jenkins LW. Neurochemical mechanisms of mild and moderate head injury: implications for treatment. In: Levin HS, Eisenberg HM, Benton AL, (Eds). Mild Head Injury. Oxford: Oxford University Press, 1989; 54-79.

20. Gurdjian ES. Recent advances in the study of the mechanism of impact injury of the head: a summary. Clin Neurosurg 1972; 19: $1-42$.

21. Pellman EJ. Commentary: Background on the National Football League's research on concussion in professional football. Neurosurgery 2003; 53:797-798.

22. Pellman EJ, Viano DC, Tucker AM, Casson IR, Waeckerle JF. Concussion in professional football: reconstruction of game impacts and injuries. Neurosurgery 2003; 53:799-814.

23. Pellman EJ, Viano DC, Tucker AM, Casson IR. Concussion in professional football: location and direction of helmet impacts Part 2. Neurosurgery 2003; 53:1328-1341. 